\title{
Sub aperture polishing of Fused Silica asphere for deterministic control of form and texture
}

\author{
*Neeraj Pandey, Anil Kumar, K. K. Pant, A. Ghosh \\ Instruments Research \& Development Establishment, \\ Dehradun, India-248008 \\ neerajpandey@irde.drdo.in, neerajspn@gmail.com
}

\begin{abstract}
Aspheric lenses are the lenses, which have varying radius of curvature according to distance from the optical axis. Optical designer can do excellent aberration correction using an aspheric lens, which provides superior resolution, even with compact optics composed of a small number of elements. We report fabrication of an aspheric lens in Fused Silica using sub aperture polishing technique as traditional full aperture polishing is not suitable for aspheric lenses. The linear feed rate has been optimized to produce optical quality surface finish using less number of iterations. Stylus profilometer and optical profilometer has been used for aspheric surface characterization.
\end{abstract}

Keywords - Aspheric Optics, Sub aperture polishing, Optical fabrication.

\section{INTRODUCTION}

A lens is an important part of any optical system. These lenses may have spherical, aspheric or diffractive surface. As per the shape of the lens, fabrication methods are different. A spherical surface is easy to fabricate using conventional grinding and polishing machines [1]. Also, the spherical surfaces are easy to measure using a Newton interferometer setup [2]. The fabrication methods and measurement systems for aspheric and diffractive optics are quite difficult and challenging.

Spherical surfaces are not usually the optimal shape for lens surfaces or for mirrors for most optical applications. Optical systems can benefit tremendously, if they can use aspherical surfaces - surfaces that are not spherical. The use of aspheres allows better quality images with the use of fewer elements. The application of aspheric surfaces is limited to a tiny fraction of optics because of the difficulty in fabricating and testing these surfaces. Optical designers always require less number of surfaces with more degree of freedom for removing the aberrations. Using aspheric surfaces, optical designer can design an optical system with less number of elements, small optical system and improved performance [3]. Spherical surface has only one radius of curvature. It is easy to fabricate using classical grinding \& polishing techniques.
Aspheric surface has variation in radius of curvature along different zone.

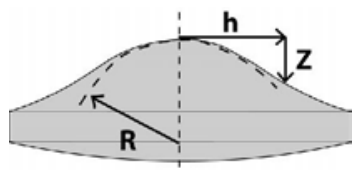

Figure1. Aspheric Surface

The sag equation of an aspheric surface is as following:

$$
z \frac{c h^{2}}{1 \sqrt{1(1 k) c^{2} h^{2}}}{ }^{A h^{4}}{ }^{B h^{\circ}}
$$

Where, $\mathrm{c}$ is the curvature $=(1 / \mathrm{R}), \mathrm{k}$ denotes the conic constant $\&$ A, B, C, D are $4^{\text {th }}, 6^{\text {th }}, 8^{\text {th }}$ and $10^{\text {th }}$ order deformation coefficients respectively [4].

In this paper, we present the polishing of Fused Silica aspheric surface using sub aperture polishing technique. Aspheric optics in Fused Silica material having base radius of curvature $73.117 \mathrm{~mm}$ and diameter $12 \mathrm{~mm}$ was required for a Laser altitude sensor. Conventional all spherical design requires 3 lenses, while Aspheric design requires only single aspheric lens. Lenses were polished using sub aperture polishing method. Surface measurements were done using stylus profilometer and optical profilometer.

The component details are as following:

Material - Fused Silica

Final edge diameter $=12.000 \mathrm{~mm}$

Spherical radius $\mathrm{R} 1=10.711 \mathrm{~mm}$ Convex

Aspheric base radius R2 $=73.117 \mathrm{~mm}$ Convex 
Conic constant $\mathrm{k}=$

0 Aspheric terms:

$\mathrm{A}=0.103578 \mathrm{e}-03, \mathrm{~B}=-0.213284 \mathrm{e}-06$

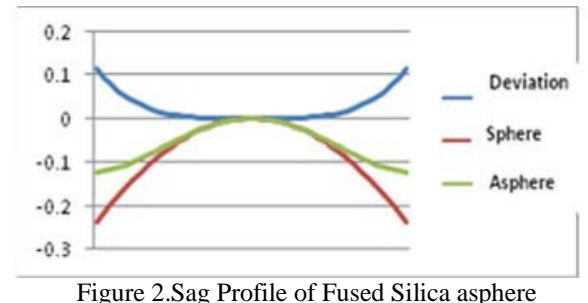

Figure 2 shows the sag profile analysis. The red, green and blue curves show the sphere sag, asphere sag and the departure of asphere from base sphere respectively. The maximum departure at the edge is $50 \mathrm{~m}$. The sag analysis shows that the surface is convex at the center while it is being concave at the edges. The curvature is changing form center to edge and there is inflection point. This behavior of asphere creates problem in polishing as well as in testing of surface.

\section{SUB APERTURE POLISHING TECHNIQUE}

Full aperture technique is best suited for spherical optics, while an aspheric surface has zonal departures in various zones. A full aperture polishing tool cannot correct aspheric surface therefore using a smaller sub aperture tool, we will get finer corrections over the various zones [5]. The main difference between full aperture polishing and sub aperture polishing is that only a small section of the aperture is in contact with the polishing tool in sub aperture polishing.

Classical full aperture polishing method uses pitch or polyurethane pad for polisher. We cannot vary the polishing spot in full aperture polishing. The spot size of a membrane based sub aperture tool can be controlled by the application of pressure.

In sub aperture polishing, the tool and polishing medium selection is driven by the localized radius of asphere as well as the material properties. We used polyurethane (LP66) and Uninap cloth for various polishing cycles [6].

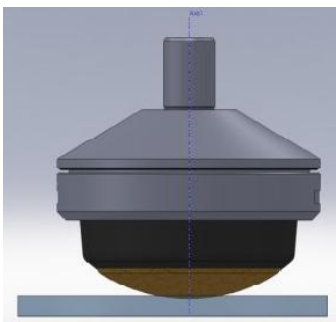

Figure 3. (a) Sub aperture tool

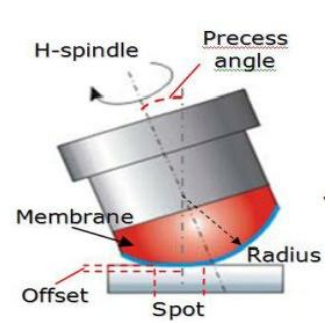

(b) Sub aperture tool geometry constant $\left(c_{p}\right)$ describes molecular level effects during the

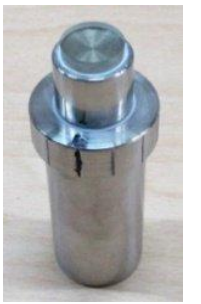

Figure 4. (a) Mounting tool

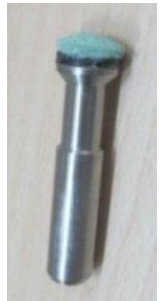

(b) Polishing tool
Sub aperture tool geometry is shown in figure 3(b). We have developed a mounting tool in stainless steel material for easy mounting of aspheric lens and to remove centering error problem due to mounting. A sub aperture polishing tool is shown in figure 4(b).

\section{GRINDING OF ASPHERICS SURFACE}

First, both the surfaces were grinded flat and edging of the part was done up to $14 \mathrm{~mm}$ diameter. Aspheric surface was generated using diamond cup tools having different grit sizesD46, D17, D6. For aspheric surface, first a best fit radius was generated to reduce the excess material removal. In the figure 5 yellow, green and red curve show asphere sag, best fit sphere and the deviation of best fit sphere respectively

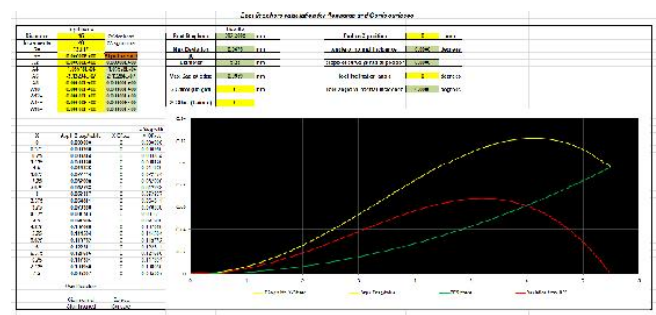

Figure 5. Best fit radius calculation

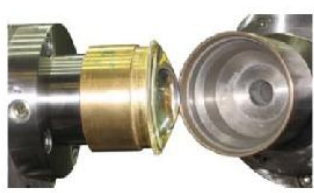

Figure 6. (a) Cup tool grinding

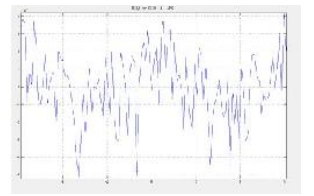

(b) Grinded surface profile -Peak to Valley (PV) $1.5 \mathrm{~m}$

\section{POLISHING, MEASUREMENT \& SUCCESSIVE ITERATIONS}

Material removal rate during the optical polishing can be defined by the Preston's equation [7] and it depends up on the tool pressure and relative velocity between tool and the lens surface.

$$
\frac{d m}{d t} C \underset{p 0 r}{P V}
$$

where $\mathrm{dm} / \mathrm{dt}$ is the average thickness removal rate, $\mathrm{P}_{0}$ is the applied pressure, and $\mathrm{V}_{\mathrm{r}}$ is the average relative velocity of the polishing particle relative to the substrate. The Preston's polishing process. The rate of removal increases linearly with 
pressure and velocity. By changing the relative velocity between polishing tool and substrate, one can achieve desired material removal at the substrate. In our method of sub aperture polishing, we have described material removal in terms of variable linear feed rate over the part and dwell time approach has been applied for material removal [8].

We have used raster polishing mode for polishing of Fused Silica aspheric surface for local corrections. In raster polishing mode, job axis (C-Axis) is stationary while tool is moving either along $\mathrm{X}$-axis or $\mathrm{Y}$-axis. During raster polishing, feed rate is variable as per the error profile of the surface. Hence material removal rate is different in different regions of surface and we get desired material removal at surface along different regions.

First, we have done polishing trials on a Fused Silica flat surface having diameter $25 \mathrm{~mm}$. The flat surface has been polished using different feed rates in seven polishing iterations. The roughness of the flat surface has been measured after each polishing cycle. It was observed that a lower value of feed rate gives better polishing quality.

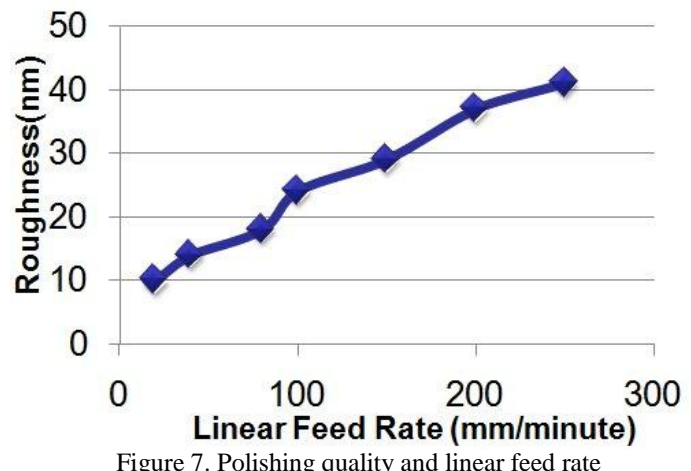

The polishing time at different feed rates is also monitored. The lower feed rate polishing cycle takes much time. So there is a trade of between polishing quality and polishing time. As per the required surface accuracy, the linear feed rate and polishing time has been optimized for the polishing for fused silica asphere.

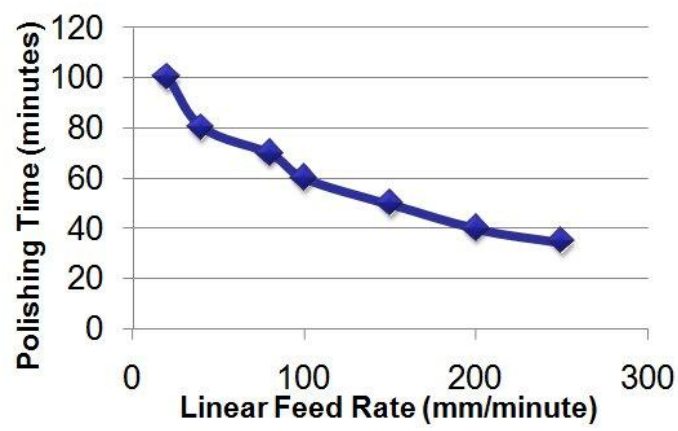

Figure 8. Polishing time and linear feed rate
Polyurethane pad is used for pre-polishing due to fast cutting action. The polishing tool is dressed after two polishing runs and Uninap cloth has been used in final polishing runs for surface roughness improvements. The polishing medium depends upon the material to be polished. For optical glasses, Cerium Oxide is the choice of material as polishing medium [9]. Cerium Oxide having particle size 1 micron with deionized water is used in polishing slurry. The slurry density was maintained $1.3 \mathrm{gm} / \mathrm{cm} 3$ and room temperature was maintained at $24{ }^{0} \mathrm{C}$.

Raster mode has been chosen over the sync-spiral polishing mode because grinded surface is very good and surface peak to valley (PV) is of the order of $1.5 \mathrm{~m}$. Surface profile measurements were done using Form Talysurf contact profilometer. Linear feed rate has been optimized as per the surface profile measurements and required corrections. Feed rate values between $20 \mathrm{~mm} /$ minute to $80 \mathrm{~mm} /$ minute provide good surface finish. Feed rate values more than 80 $\mathrm{mm} /$ minute degrade the surface while feed rate values less than $20 \mathrm{~mm} / \mathrm{minute}$ takes longer polishing time. The feed rate is moderated as per the required material removal at a particular place.

Figures 9 to 13 show the feed rate profiles and surface profiles in various polishing cycles. After first polishing cycle, the measured surface profile is shown in figure 10(a). In this surface profile, more material has been removed at the central part as compared to material removed at the outer zonal part. In next polishing cycle, feed rate (figure 10b) is moderated such that high feed rate is at central part while lower feed rate at the outer zonal part. Hence material removal rate is small at the central part while it is more at the outer zonal part. Using this approach to moderate linear feed rate, the surface peak to valley (PV) was reduced in successive polishing iterations.

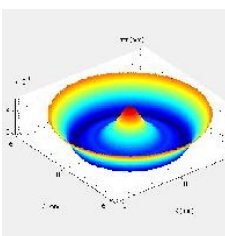

(a)

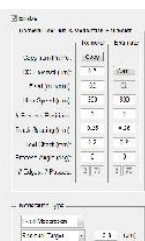

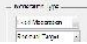

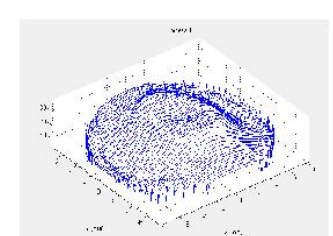

(b)

Figure 9. (a) Initial Surface profile (PV-1.5 m) after grinding (b) Feed rate in Ist polishing cycle

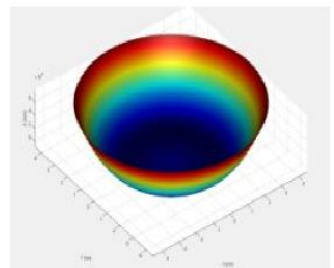

(a)

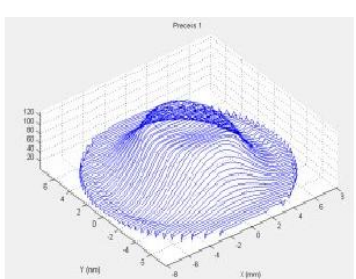

(b)

Figure 10. (a) Surface profile (PV-1.3 m) after Ist polishing cycle (b) Feed rate in Ind polishing cycle 


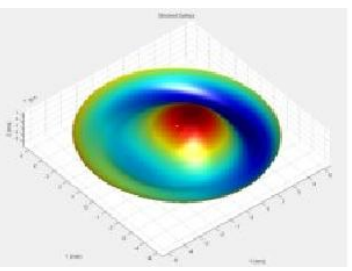

(a)

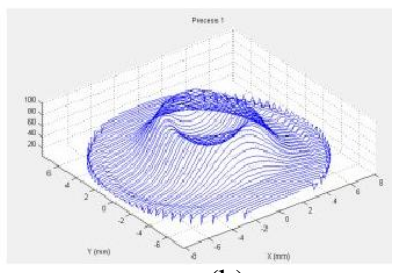

(b)
Figure 11. (a) Surface profile (PV-1 m) after IInd polishing cycle (b) Feed rate in IIIrd polishing cycle

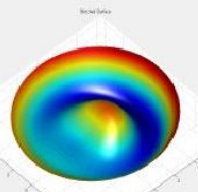

(a)

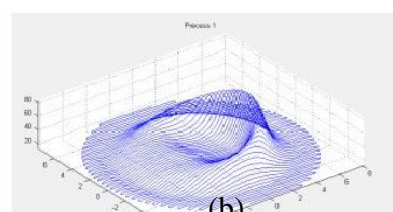

(b)
Figure 12. (a) Surface profile (PV-0.8 m) after IIIrd polishing cycle (b) Feed rate in IVth polishing cycle

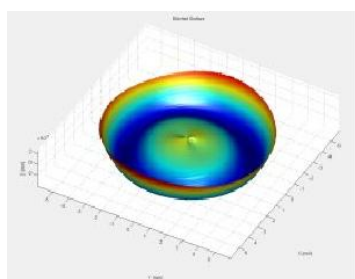

(a)

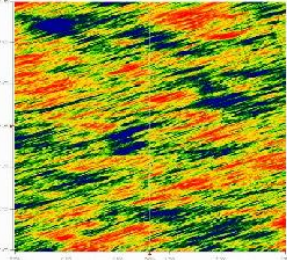

(b)
Figure 13. (a) Surface profile (PV-0.4 m) after IVth polishing cycle (b) Surface roughness $10 \mathrm{~nm}$ after IVth polishing cycle

Among four polishing runs, first two runs are raster with polyurethane LP 66 pad having tool radius $14 \mathrm{~mm}$, while other two raster runs with Uninap cloth having tool radius 7 $\mathrm{mm}$. In four consecutive polishing iterations, we achieve successive improvements on surface and finally we achieved surface peak to valley (PV) $0.4 \mathrm{~m}$ and roughness (Ra): $10 \mathrm{~nm}$.

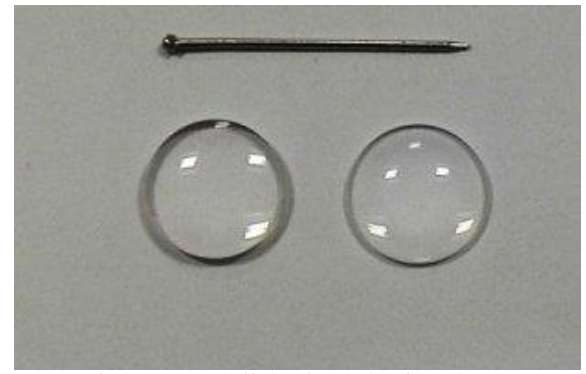

Figure 14. Polished Aspheric Lenses

\section{CONCLUSION}

Convex aspheric surface in fused silica material has been polished successfully using sub aperture polishing technique. The linear feed rate has been optimized for better results and surface accuracy. We have done four polishing runs having total polishing time 147 minutes. The finished surface has peak to valley (PV): $0.4 \mathrm{~m}$, RMS: $40 \mathrm{~nm}$ and roughness (Ra): $10 \mathrm{~nm}$.

Sub aperture polishing is a powerful tool that can be used to finish aspheres without the need for artisan techniques or skilled operators. Sub aperture polishing technique has enabled aspheres to be produced in a cost effective and deterministic manner with significantly reduced set up times, when compared with the traditional polishing techniques.

\section{ACKNOWLEDGEMENT}

Authors are thankful to Dr. S. S. Negi, Director, IRDE for his support and encouragement for the work. Authors are also thankful to Sh. S. P. Gaba, Scientist-'G', for his valuable suggestions.

\section{REFERENCES}

[1] Geoffrey Boothroyd, [Handbook of Lapping and Polishing], CRC Press, Taylor \& Francis Group, New York 2007.

[2] M. V. Mantravadi, Newton, Fizeau, and Haidinger Interferometers, [Optical Shop Testing], Third Edition, John Wiley \& Sons, Inc., New Jearsy 2007.

[3] B. Braunecker, R. Hentschel, and H.J. Tiziani, [Advanced Optics Using Aspherical Elements], SPIE ebook, 2008.

[4] J. Kumler, "Designing and Specifying Aspheres for Manufacturability”, Jenoptik Optical Systems, Inc.

[5] Neeraj Pandey, "Pitch polishing of semiconductor optical materials using continuous iterative interferrogram analysis" [Physics of Semiconductor Devices], Proc. IWPSD-2013, 10-13, Dec 2013, India, Springer International Publishing, 2014, pp 811-812.

[6] Marc Tricard, "Subaperture approaches for asphere polishing and metrology”, Proc. SPIE 5638, Optical Design and Testing II, 284 (April 06, 2005).

[7] F. W. Preston, "The Theory and Design of Plate Glass Polishing Machines” J. Soc. Glass Tech. 11, 214, 1927.

[8] R G Bingham, “A Novel Automated Process for Aspheric Surfaces”, Proc. SPIE 45th Annual Meeting, 2000, Vol. 4093 'Current Developments in Lens Optical Design and Engineering'; pp445-448.

[9] L. M. Cook, “Chemical processes in glass polishing” Journal of NonCrystalline Solids Volume 120, Issues 1-3, 1 April 1990, Pages 152171. 